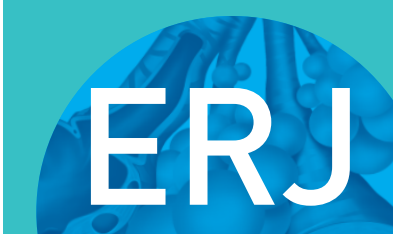

open research
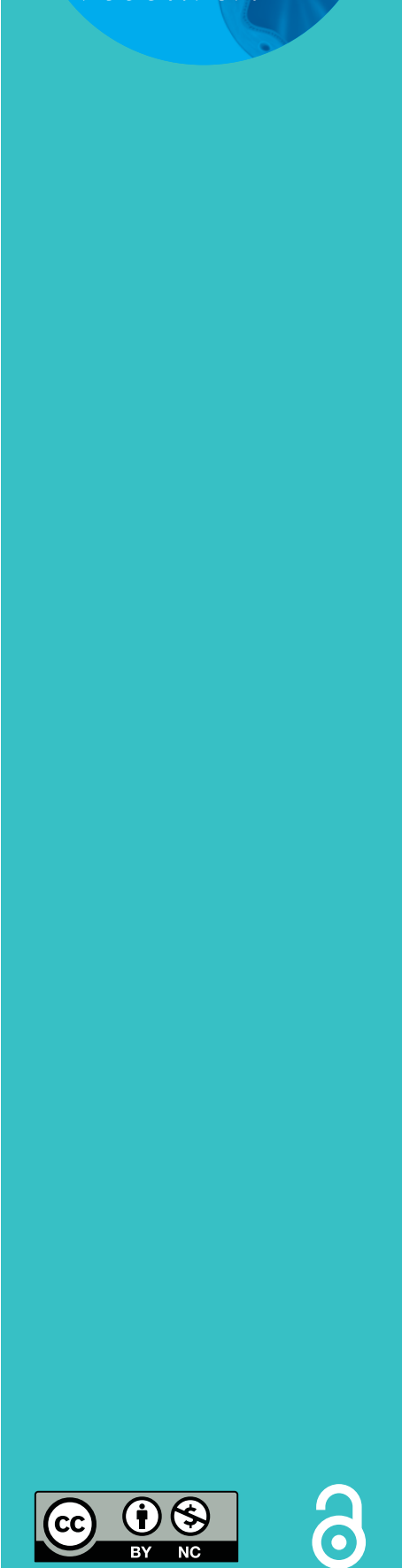

\section{Dose-response characteristics of noninvasive ventilation in acute respiratory failure}

\author{
Craig Hukins (1), Michelle Murphy and Timothy Edwards
}

Affiliation: Dept of Respiratory and Sleep Medicine, Princess Alexandra Hospital, Woolloongabba, Australia.

Correspondence: Craig Hukins, Dept of Respiratory and Sleep Medicine, Princess Alexandra Hospital, Ipswich Road, Woolloongabba, Queensland 4102, Australia. E-mail: craig.hukins@ahealth.qld.gov.au

ABSTRACT Acute noninvasive ventilation (NIV) is a well-established therapy for acute respiratory failure but the dose-response characteristics of this therapy have not been defined. The aim of this study was to define this dose-response relationship.

This study was a retrospective review of patients receiving NIV for acute respiratory failure in a tertiary hospital respiratory high-dependency unit between July 2012 and June 2017. Mask-on time (rather than the period that NIV was in use) as the "dose" was compared with hospital survival as the "response".

654 patients were included, 594 (91\%) with hypercapnic respiratory failure (HCRF). NIV was used for a median (interquartile range (IQR)) duration of 2.74 (1.51-4.73) days and median (IQR) mask-on time was 34 (18-60) h (56.1\% (41.2-69.5\%) of treatment time). There was evidence of a dose-response relationship in the HCRF group up to a ceiling of $24 \mathrm{~h}$ mask-on time, but not in the hypoxaemic respiratory failure (HRF) group. There was a difference in survival with as little as $2 \mathrm{~h}$ mask-on time $(92 \%$ compared with $73 \%$; $\mathrm{p}<0.001$ ). Patients requiring NIV for $80-100 \%$ of therapy time had lower survival.

We conclude that there is evidence of a dose-response relationship between cumulative NIV usage (mask-on time) and survival from as little as $2 \mathrm{~h}$ to a ceiling of $\sim 24 \mathrm{~h}$ in HCRF, but not in HRF.

@ERSpublications

Acute NIV in respiratory failure has a dose-response effect on survival from as little as $\mathbf{2} \mathrm{h}$ of therapy http://bit.ly/2okErQZ

Cite this article as: Hukins C, Murphy M, Edwards T. Dose-response characteristics of noninvasive ventilation in acute respiratory failure. ERJ Open Res 2020; 6: 00041-2019 [https://doi.org/10.1183/ 23120541.00041-2019].

Received: 14 Feb 2019 | Accepted after revision: 23 Sept 2019

Copyright $\odot$ ERS 2020. This article is open access and distributed under the terms of the Creative Commons Attribution Non-Commercial Licence 4.0. 


\section{Introduction}

Noninvasive ventilation (NIV) outside of the intensive care unit (ICU) is well established as a highly effective therapy for acute respiratory failure [1,2]. The highest quality of evidence is seen in acute hypercapnic respiratory failure (HCRF) in chronic obstructive pulmonary disease (COPD) [3]. Despite consistent data showing the efficacy of NIV overall, there are very limited data on the dose-response characteristics of the therapy, especially related to therapy duration and intensity. Studies of NIV in acute respiratory failure most commonly report only the total period that therapy was offered rather than cumulative actual use (mask-on time). Where reported, the actual daily use of NIV is often only between 8 and $14 \mathrm{~h}$ in the first $24 \mathrm{~h} \mathrm{[3-7].} \mathrm{There} \mathrm{are} \mathrm{limited} \mathrm{indirect} \mathrm{data} \mathrm{supporting} \mathrm{a} \mathrm{dose-response} \mathrm{relationship} \mathrm{in}$ acute NIV. For example, more intensive NIV use (notably nocturnal NIV) was associated with better response to therapy in one study [8]. Studies on chronic nocturnal ventilation also indirectly suggest a dose-response relationship. A small number of studies in patients with motor neurone disease [9, 10], obesity hypoventilation [11] and restrictive chest wall disease [12] have suggested that survival benefits are only seen if average usage is greater than a threshold of $\geqslant 4 \mathrm{~h}$ per night.

Therefore, there is no evidence to guide this therapy, which is regarded as standard of care (in at least HCRF due to COPD) in terms of minimally effective or optimal durations of therapy. Furthermore, despite the absence of robust data on the dose-response characteristics of acute NIV, and specifically the minimal effective duration of therapy, health funding agencies utilise arbitrary thresholds in service funding models. In Australian public hospitals, for example, only acute NIV with cumulative actual usage (mask-on time) of $\geqslant 24 \mathrm{~h}$ is recognised for funding. In our institution (Princess Alexandra Hospital, Woolloongabba, Australia), 30-40\% of acute NIV does not qualify for funding under current criteria.

The aim of this study was to determine the dose-response characteristics between the duration of acute NIV and survival, and to identify if there is a threshold for minimum duration of acute NIV associated with survival benefits.

\section{Methods}

The study was a single-centre, retrospective review of patients admitted to a tertiary hospital (Princess Alexandra Hospital) respiratory high-dependency unit (HDU) over a 5-year period (between July 1, 2012 and June 30,2017). Patients requiring acute NIV for acute respiratory failure were included; however, patients stepped-down from the ICU were excluded. All data were collected prospectively and entered in an in-house database (FileMaker Pro; FileMaker, Santa Clara, CA, USA). The study was approved by the institutional Human Research Ethics Committee.

HCRF was defined as an acute elevation in arterial carbon dioxide tension $>45 \mathrm{mmHg}$ and acidosis defined as arterial $\mathrm{pH}<7.35$. Hypoxaemic respiratory failure (HRF) was defined as an acute state with arterial oxygen tension $<60 \mathrm{mmHg}$ on room air (or, where room air measurements were not available, the requirement for oxygen to maintain pulse oximetry measurements $>90 \%$ ) with normal or low partial pressure of carbon dioxide [13]. Clinical data including age, sex, active comorbidities, admission arterial blood gas results, usual functional status (World Health Organization (WHO) Functional Class) and treatment outcomes (including escalation to ICU and in-hospital mortality) were recorded.

The duration of NIV therapy (therapy time) was defined as the total period that NIV was offered (including both mask-on and mask-off periods), and was calculated as the difference (in days) between commencement and final discontinuation of NIV. Mask-on time was defined as the actual usage of the therapy (in hours) during the admission, and was calculated from the differences in the device hour-meter readings at commencement and discontinuation of therapy. Mask-on proportion was defined as the proportion of the therapy time when NIV was actually in use and was calculated as the percentage of mask-on time/therapy time. The protocol of the respiratory HDU in our institution for the management of acute respiratory failure is continuous NIV with short breaks until the respiratory failure has corrected followed by progressive weaning of wakeful then nocturnal NIV as tolerated with discontinuation of the therapy altogether once the patient is clinically stable. Out-of-protocol discontinuation was defined as the ceasing of NIV before deemed clinical stable. The reasons for this discontinuation were recorded.

\section{Outcome measures}

The co-primary outcome measures were the dose-response relationships between hospital survival and the mask-on time (reflecting therapy "dose") in terms of 1) usage bands (mask-on time <4, 4-8, 8-16, 16-24 and $>24 \mathrm{~h}$ ) to calculate dose-response characteristics and 2) usage thresholds (mask-on time $<2,<4,<8$, $<16,<24$ and $<48 \mathrm{~h}$ ) to calculate minimally effective therapy duration. Secondary outcomes were the relationship between hospital survival and mask-on proportion in bands of $20 \%(<20 \%, 20-40 \%$ and $40-60 \%)$ as a measure of therapy "intensity", and the associations between mortality and clinical factors (age, sex, active comorbidities, WHO Functional Class and arterial blood gas parameters). 


\section{Statistical analysis}

Statistical analysis was performed using SigmaPlot version 13 (Systat Software, San Jose, CA, USA). Data are presented as median (interquartile range (IQR)) as all variables were nonparametric. Continuous variables were compared by one-way ANOVA on ranks. Categorical parameters and proportions were compared using Chi-squared analysis. Multiple logistic regression analysis was used to identify clinical parameters (age, sex, ventilation duration, baseline arterial blood gas results and active comorbidities) associated with risk of death and odds ratios (95\% confidence intervals) were calculated for the risk of mortality for these parameters. Log-rank Kaplan-Meier survival analysis was used to compare survival between groups. $\mathrm{p}<0.05$ was considered significant.

\section{Results}

\section{Patients}

There were 654 patients with acute respiratory failure (594 (91\%) with HCRF) admitted to the respiratory HDU and treated with acute NIV between July 1, 2012 and June 30, 2017. Clinical data are shown in table 1 .

Patients were typically middle aged to elderly with almost equal sex distribution overall, but there was a significant male predominance in HRF. Active comorbidities differed between groups (table 1), with relatively more patients with COPD and obesity in the HCRF cohort, and more with pneumonia, lung cancer and interstitial lung disease in the HRF group. Usual functional status also differed between the groups, with relatively more HRF patients with a normal (WHO Functional Class I) pre-morbid functional status. There were also significant differences in arterial blood gas measurements between the cohorts as expected.

NIV parameters are shown in table 2. Median therapy duration with NIV (therapy days) was 2.74 days with a median mask-on time of $34 \mathrm{~h}(56.1 \%$ of therapy duration). There were no differences in these

\section{TABLE 1 Clinical characteristics of the study cohort}

\begin{tabular}{|c|c|c|c|c|}
\hline & All patients & HCRF patients & HRF patients & $p$-value \\
\hline Subjects & 654 & 594 & 60 & \\
\hline Age years & $64.3(53.4-71.9)$ & $55.2(41.5-68.5)$ & $64.9(55.3-73.9)$ & 0.79 \\
\hline Male:female (\% male) & $363: 291(55)$ & $317: 277$ (53) & $46: 14(77)$ & $<0.001$ \\
\hline \multicolumn{5}{|l|}{ Diagnoses } \\
\hline COPD & 375 (57) & $360(61)$ & $15(25)$ & $<0.001$ \\
\hline Asthma & $35(5)$ & $34(6)$ & $1(2)$ & 0.30 \\
\hline Pneumonia & $117(18)$ & $88(15)$ & $29(48)$ & $<0.001$ \\
\hline LRTI & $22(3)$ & $20(3)$ & $2(3)$ & 0.72 \\
\hline Bronchiectasis & $27(4)$ & $26(4)$ & $1(2)$ & 0.51 \\
\hline Lung cancer & $25(3)$ & $16(3)$ & $9(15)$ & $<0.001$ \\
\hline Interstitial lung disease & $15(2)$ & $4(<1)$ & $11(18)$ & $<0.001$ \\
\hline Acute pulmonary oedema & $41(6)$ & $35(6)$ & $6(10)$ & 0.33 \\
\hline Sleep disordered breathing & $171(26)$ & $158(27)$ & $13(22)$ & 0.50 \\
\hline Obesity & 243 (37) & $231(31)$ & $12(20)$ & 0.006 \\
\hline Motor neurone disease & $23(4)$ & $21(4)$ & 2 (3) & 0.77 \\
\hline \multicolumn{5}{|l|}{ Admission gas exchange } \\
\hline $\mathrm{pH}$ & $7.43(7.29-7.40)$ & 7.34 (7.29-7.39) & $7.44(7.39-7.47)$ & $<0.001$ \\
\hline$P_{\mathrm{aCO}_{2}} \mathrm{mmHg}$ & $62.1(53.0-75.0)$ & $65.0(55.0-76.0)$ & $38.0(32.5-43.0)$ & $<0.001$ \\
\hline$P_{\mathrm{aO}_{2}} / F_{\mathrm{IO}_{2}}$ & $220.0(180.0-258.9)$ & $222.7(185.7-261.9)$ & $147.2(79.0-244.5)$ & $<0.001$ \\
\hline Mortality & $68(10.4)$ & $47(7.9)$ & $21(35.0)$ & $<0.001$ \\
\hline Patients requiring ICU & $12(2)$ & $5(<1)$ & $7(12)$ & $<0.001$ \\
\hline Subsequent mortality & $5(42)$ & $1(20)$ & $4(57)$ & 0.30 \\
\hline \multicolumn{5}{|l|}{ Usual WHO Functional Class } \\
\hline । & $43(7)$ & $32(5)$ & $11(18)$ & \\
\hline II & $311(48)$ & $284(48)$ & $27(45)$ & $<0.001$ \\
\hline III & $298(46)$ & 188 (32) & $10(17)$ & \\
\hline IV & 98 (15) & $86(15)$ & $12(20)$ & \\
\hline
\end{tabular}

Data are presented as $\mathrm{n}$, median (interquartile range) or $\mathrm{n}(\%)$, unless otherwise stated. HCRF: hypercapnic respiratory failure; HRF: hypoxaemic respiratory failure; COPD: chronic obstructive pulmonary disease; LRTI: lower respiratory tract infection; $P_{\mathrm{acO}_{2}}$ : arterial carbon dioxide tension; $P_{\mathrm{aO}}$ : arterial oxygen tension; $F_{\mathrm{IO}_{2}}$ : inspiratory oxygen fraction; ICU: intensive care unit; WHO: World Health Organization. p-values refer to differences between HCRF and HRF cohorts. 


\begin{tabular}{|c|c|c|c|c|}
\hline & All patients & HCRF patients & HRF patients & p-value \\
\hline Subjects & 654 & 594 & 60 & \\
\hline Duration of NIV therapy ${ }^{\#}$ days & $2.74(1.51-4.73)$ & $2.76(1.53-1.23)$ & $2.25(1.23-5.95)$ & 0.56 \\
\hline Total mask-on time ${ }^{\pi} h$ & $34.0(18.0-60.0)$ & $34.0(18.2-60.0)$ & $34.0(10.0-60.0)$ & 0.42 \\
\hline Proportion of mask-on time $\%$ & $56.1(41.2-69.5)$ & $55.2(41.5-68.5)$ & $65.6(33.7-85.2)$ & 0.07 \\
\hline \multicolumn{5}{|l|}{ Settings } \\
\hline Inspiratory pressure $\mathrm{cmH}_{2} \mathrm{O}$ & $18.0(16.0-22.0)$ & $19.0(16.0-22.0)$ & $15.0(12.5-16.8)$ & $<0.001$ \\
\hline Expiratory pressure $\mathrm{cmH}_{2} \mathrm{O}$ & $10(8-14)$ & $10(8-14)$ & $8.5(6.5-10.0)$ & 0.07 \\
\hline Pressure support $\mathrm{cmH}_{2} \mathrm{O}$ & $8(5-15)$ & $8(5-15)$ & $6(4-11)$ & $<0.001$ \\
\hline Out-of-protocol cessation of $\mathrm{NIV}^{+}$ & $37(6)$ & $29(4)$ & $8(13)$ & 0.02 \\
\hline Intolerance of treatment & 20 & 18 & 2 & \\
\hline Change to palliative treatment intent & 12 & 7 & 5 & 0.10 \\
\hline Treatment ineffective & 5 & 4 & 1 & \\
\hline \multicolumn{5}{|c|}{$\begin{array}{l}\text { Data are presented as } n \text {, median (interquartile range) or } n(\%) \text {, unless otherwise stated. HCRF: } \\
\text { hypercapnic respiratory failure; HRF: hypoxaemic respiratory failure. }{ }^{\prime} \text { : overall period where NIV was used } \\
\text { lincluding mask-on and mask-off durations); }{ }^{\uparrow} \text { : cumulative period where the therapy was actually in use; } \\
{ }^{+} \text {: therapy was stopped before clinical stability was achieved. } p \text {-values refer to differences between HCRF } \\
\text { and HRF cohorts. }\end{array}$} \\
\hline
\end{tabular}

measures between HCRF. Inspiratory pressure and pressure support (the difference between inspiratory and expiratory pressures) were higher in the HCRF cohort (both $\mathrm{p}<0.001$ ), but there was no difference in expiratory pressure. Out-of-protocol (before clinically stable) cessation of NIV was more common in HRF ( $13 \%$ compared with $4 \% ; \mathrm{p}=0.016$ ), although the reasons for the cessation were similar between groups (table 2).

\section{In-hospital survival}

Overall in-hospital mortality was $10.4 \%$ (7.9\% in HCRF and $35.0 \%$ in HRF; p $<0.001$ ) (table 1). Survival was significantly lower in the HRF cohort. Clinical factors associated with an increased risk of death were active diagnoses of interstitial lung disease (OR 11.1 (95\% CI 2.5-48.6); p<0.001) and lung cancer (OR 7.1 (95\% CI 2.0-24.9); $\mathrm{p}=0.002)$. Obesity was associated with a reduced risk of death (OR 0.2 (95\% CI $0.1-0.5) ; \mathrm{p}<0.001)$.

\section{Relationships between survival and mask-on time}

Mask-on duration, i.e. the cumulative duration of actual use of mask ventilation, reflects the "dose" of NIV.

There was a dose-response relationship between survival and mask-on time (represented in bands of cumulative usage $<4,4-8,8-16,16-24$ and $>24 h)$ in the whole group $(p<0.001)$, which was evident in only the HCRF cohort $(\mathrm{p}<0.001)$ and not the HRF group $(\mathrm{p}=0.55)$ (figure 1$)$. There was no difference between 16 and $24 \mathrm{~h}$ and $>24 \mathrm{~h}$ mask-on time in both the whole cohort and HCRF patients, suggesting a ceiling effect in the dose-response relationship.

There was also evidence of a dose-response relationship between survival and thresholds of mask-on time across a wider range of mask-on times $(<2,<4,<8,<16,<24$ and $<48 \mathrm{~h}$ ) (figure 2). The relationship was seen in the HCRF group across the range of thresholds from 4 to $24 \mathrm{~h}$ mask-on time $(\mathrm{p}=0.01)$, but was not present in the HRF group ( $p=0.73$ ). There appeared to be incremental improvement in survival in the HCRF group above the threshold of $2 \mathrm{~h}$ mask-on time in the entire group and above $4 \mathrm{~h}$ mask-on time in the HCRF group. Survival was significantly better above mask-on times thresholds of $2 \mathrm{~h}$ (OR of death with mask-on time $<2$ versus $\geqslant 2 \mathrm{~h}$ of 4.11 (95\% CI 1.64-10.28); $\mathrm{p}=0.004), 4 \mathrm{~h}$ (OR 4.66 (95\% CI 2.23-9.73); $\mathrm{p}<0.001)$, $8 \mathrm{~h}$ (OR 5.00 (95\% CI 2.55-9.42); $\mathrm{p}<0.001)$ and $24 \mathrm{~h}$ (OR 3.23 (95\% CI 1.75-5.93); $\mathrm{p}<0.001$ ) (figure 3 ) There was no difference in survival between mask-on time $\geqslant 48$ versus $<48 \mathrm{~h}$ (OR 0.42 (95\% CI 0.16-1.08); $\mathrm{p}=0.09$ ).

\section{Relationships between survival and mask-on proportion}

Mask-on proportion, i.e. the percentage of mask-on time/total therapy time, was used to indicate NIV "intensity".

There were significant differences in survival depending on the mask-on proportion (figure 4). There was significantly lower survival in patients with mask-on for $>80 \%$ of therapy time. There was an inverse 

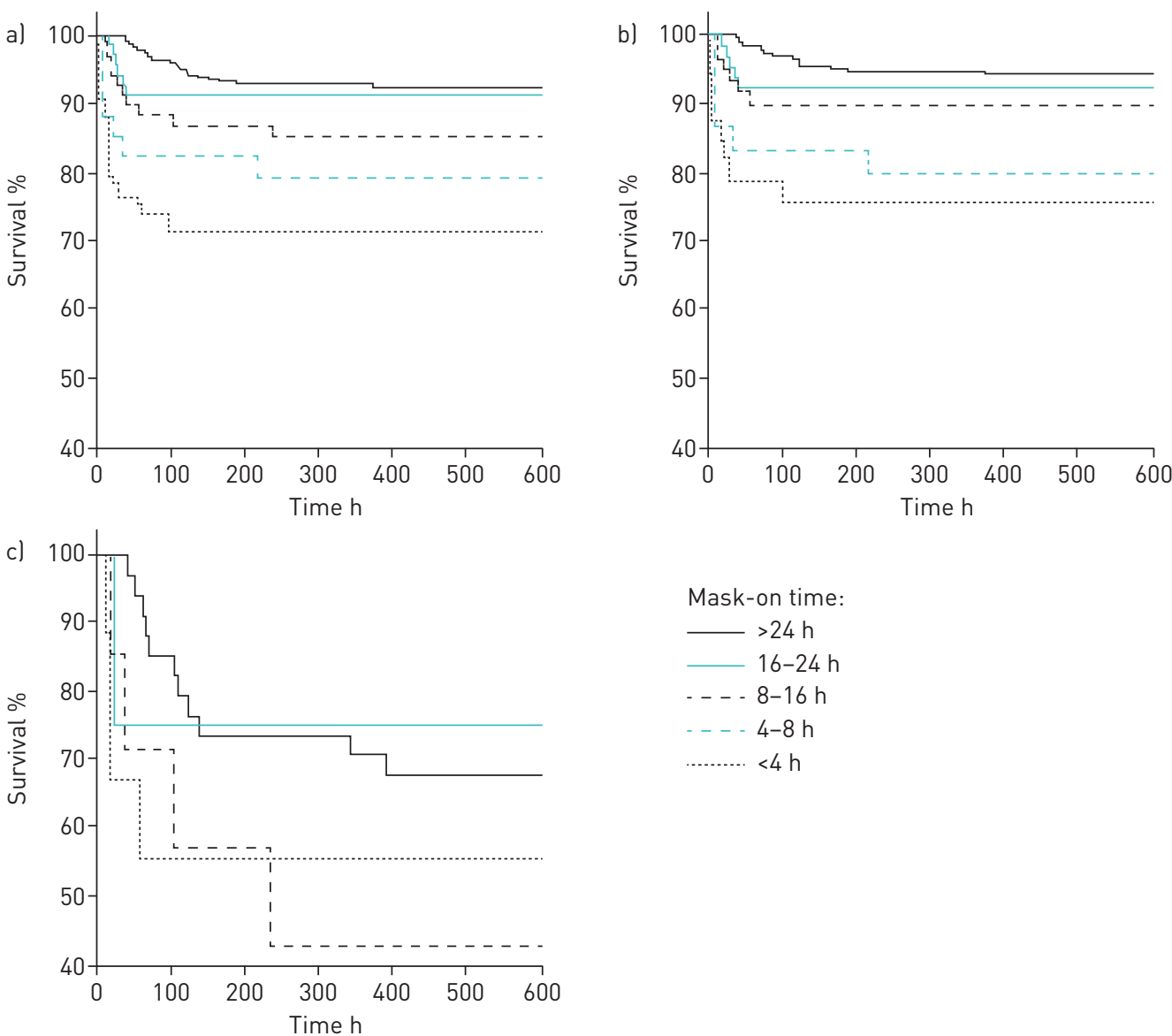

FIGURE 1 Survival stratified by type of respiratory failure and duration of mask-on time (noninvasive ventilation "dose") in bands of $\langle 4,4-8,8-16,16-24$ and $>24 \mathrm{~h}$. a) All patients, b) hypercapnic respiratory failure patients and $\mathrm{c}$ ) hypoxaemic respiratory failure patients.

dose-response relationship in the HCRF cohort, with lower proportion of use associated with better survival. No patients with HCRF died where mask-on proportion was $<20 \%$. There were significant differences between mask-on proportions with HRF $(\mathrm{p}<0.05)$, with worst survival seen in patients with mask-on proportions of $80-100 \%$, but there was no dose-response pattern. The mask-on proportion was significantly associated with admission WHO Functional Class (median (IQR): Class I $48.1 \%$ (39.6-65.4\%), Class II 47.8\% (39.5-64.2\%), Class III $55.7 \%$ (40.5-65.5\%) and Class IV $57.1 \%$ (43.2-72.1\%); $\mathrm{p}<0.001)$. However, the differences in survival in the entire cohort remained significant after correction for functional status $(\mathrm{p}<0.001)$.

\section{Discussion}

NIV is the standard of care for acute HCRF in COPD, but to the best of our knowledge this is the first study to specifically assess the dose-response characteristics of this therapy. This study is also the first to accurately report mask-on time as the primary outcome rather than just total period available for use of NIV. Median mask-on time in this study was $34 \mathrm{~h}$. NIV was utilised (mask-on) for $56 \%$ of the total therapy period. This was similar between the HCRF and HRF cohorts, although there was a trend for slightly greater proportion of use in HRF at $66 \%$ of therapy time. This usage is difficult to directly compare with other studies, but many studies have reported mask-on usage of between 6 and $8 \mathrm{~h}$ per day in the first $48 \mathrm{~h}$ of therapy $[3,4,14,15]$. The median daily usage in our patients was $>13 \mathrm{~h}$. Inspiratory pressure was slightly higher in the HCRF cohort (19 versus $15 \mathrm{cmH}_{2} \mathrm{O}$ ), but expiratory pressures were similar. Higher pressure support was therefore used in HCRF ( 8 versus $6 \mathrm{cmH}_{2} \mathrm{O}$ ), which is an expected finding as pressure support is predominantly responsible for augmenting tidal volume and alveolar ventilation, therefore reducing arterial carbon dioxide levels.

There was a reasonably consistent dose-response pattern when considering bands of mask-on time $(<4$, $4-8,8-16,16-24$ and $>24 \mathrm{~h})$ or thresholds of mask-on time $(<2,<4,<8,<16,<24$ and $<48 \mathrm{~h})$. These 

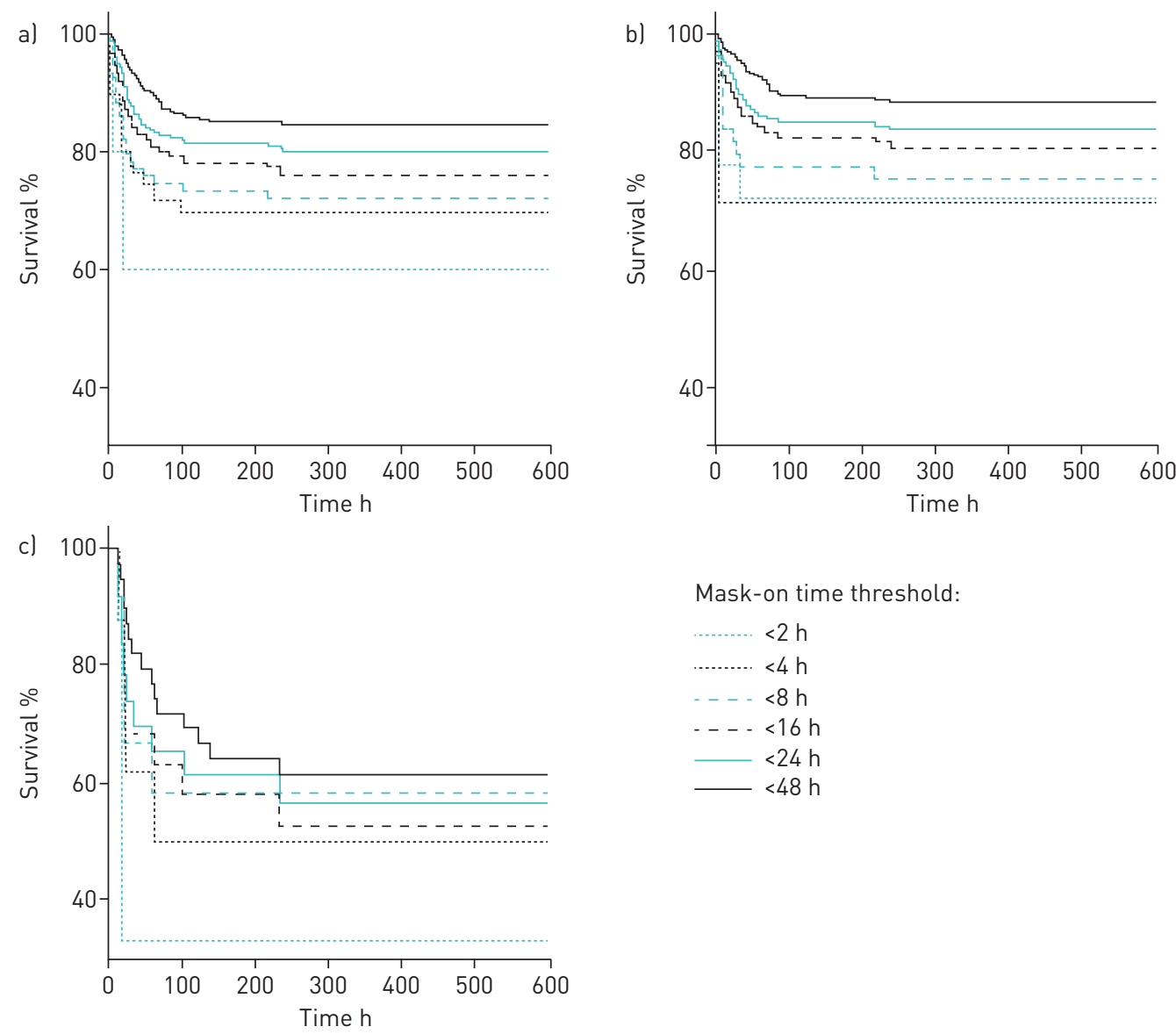

FIGURE 2 Survival stratified by type of respiratory failure and mask-on time thresholds of $<2,<4,<8,<16,<24$ and $<48 \mathrm{~h}$. a) All patients, b) hypercapnic respiratory failure patients and c) hypoxaemic respiratory failure patients.

dose-response relationships were seen only in the patients with HCRF and not those with HRF. The patterns seen in the overall group were heavily influenced by those seen in the HCRF cohort as these patients represented $>90 \%$ of all patients. The whole group data suggest a ceiling effect at $24 \mathrm{~h}$ mask-on time (i.e. survival did not improve with higher usage). The evidence for this ceiling effect at $24 \mathrm{~h}$ was: 1) the absence of clinically significant differences in survival between mask-on times $16-24$ and $>24 \mathrm{~h}$, and 2) the significant differences in survival between a mask-on time of $<24$ versus $\geqslant 24 \mathrm{~h}$, but no difference in survival between mask-on times of $<48$ versus $\geqslant 48 \mathrm{~h}$. There was a less clear lower threshold of the dose-response relationship. The data show that as little as $2 \mathrm{~h}$ mask-on time was associated with significantly lower mortality ( $7 \%$ versus $26 \%$; $<<0.001$ ). There appears to be a stepwise improvement in survival with increased usage (figure $3 a$ ). There were only 10 patients altogether with a total mask-on time of $<2 \mathrm{~h}$, which may have affected the ability to identify a lower threshold of effect.

There was evidence of an inverse dose-response relationship between survival and the mask-on proportion in the HCRF cohort $(\mathrm{p}<0.001)$. Survival was lowest in patients with a mask-on proportion of $80-100 \%$ of NIV therapy time and best in patients with a mask-on proportion of $<20 \%$ (where there were no deaths recorded). There were also significant differences in survival in the HRF cohort $(p<0.05)$, with worst survival seen in the patients with a mask-on proportion of $80-100 \%$ of therapy time. However, there was no other dose-response relationship in the HRF cohort. Mask-on proportion was associated with more impaired WHO Functional Class on admission. The association between higher mask-on proportion and worse survival is most likely explained by the covariate of higher patient acuity and increased ventilator dependence. To the best of our knowledge, the relationship between survival and intensity of NIV use (reflected by the mask-on proportion) has not been previously reported.

Mortality overall was $10.4 \%$ (7.9\% in the HCRF group and $35.0 \%$ in the HRF cohort), which is similar to other published data $[3,16,17]$. We observed significant associations between active comorbidities and survival. Others have also described lower survival in patients with interstitial lung disease [18] or lung malignancy [19] and improved survival in obesity [20]. 
a)
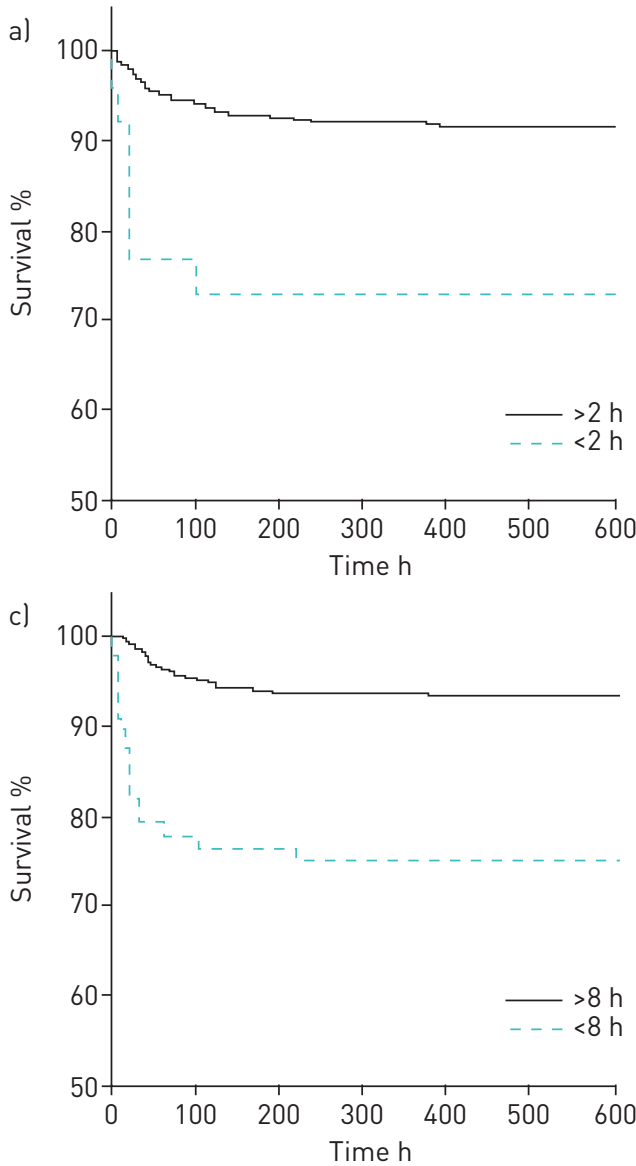

b)

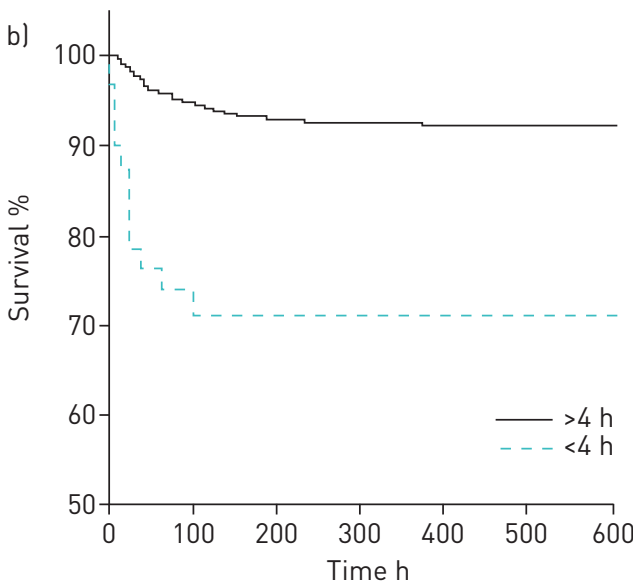

d)

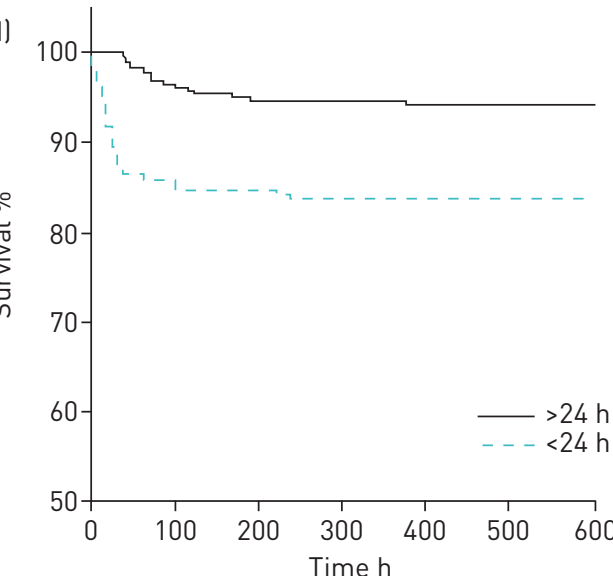

FIGURE 3 Survival stratified by threshold of mask-on time. a) $>2$ versus $<2 \mathrm{~h}$, b) $>4$ versus $<4 \mathrm{~h}, \mathrm{c}$ ) $>8$ versus $<8 \mathrm{~h}$ and $\mathrm{d})>24$ versus $<24 \mathrm{~h}$.

The study has a number of strengths and potential limitations. The size of the cohort is a strength of the study (654 patients). The recording of mask-on time based on an objective calculation from the hour-meter of the ventilator rather than observation is also a strength due to reduced risk of inaccurate measurements. The relatively small number of patients with HRF (reflecting the "real-world" incidence of respiratory failure through a respiratory HDU) is a limitation as the overall outcomes were mostly influenced by the HCRF cohort. However, the outcomes at least suggest that HCRF and HRF have different acute NIV dose-response characteristics. The main limitation of the study, as a retrospective cohort study, is that it can identify associations but not causality of the associations. Our data therefore cannot be used to determine if the differences in the duration and intensity of NIV are responsible for the observed differences in mortality, as a confounding factor may influence mortality and both duration and proportion of therapy use. Previous studies have highlighted that more rapid clinical improvement with NIV predicts better outcome $[4,21,22]$. In our case, the association between lower proportions of NIV use and improved survival may reflect a subgroup of patients with more rapid (spontaneous) clinical improvement and who were less "dependent" on ventilatory support for survival.

An understanding of the dose-response characteristics of acute NIV for acute respiratory failure potentially benefits the field in a number of respects. The demonstration of a dose-response relationship between the 2-4 and $24 \mathrm{~h}$ mask-on periods can be used together with clinical markers to help determine optimal timing for weaning of therapy. Identifying patients requiring significant proportion of times with ventilatory support $(80-100 \%$ of therapy time based on our data) as a high risk for mortality may influence treatment decisions, including the need to escalate care to an ICU if appropriate. Finally, this study provides a more robust indication of effective therapy duration to inform agencies responsible for funding of care. Our data suggest that even $2 \mathrm{~h}$ of mask-on time influences survival and would be a more appropriate threshold for funding. In Australia, government funding for acute NIV is only provided for cumulative treatment of at least $24 \mathrm{~h}$. 
a)
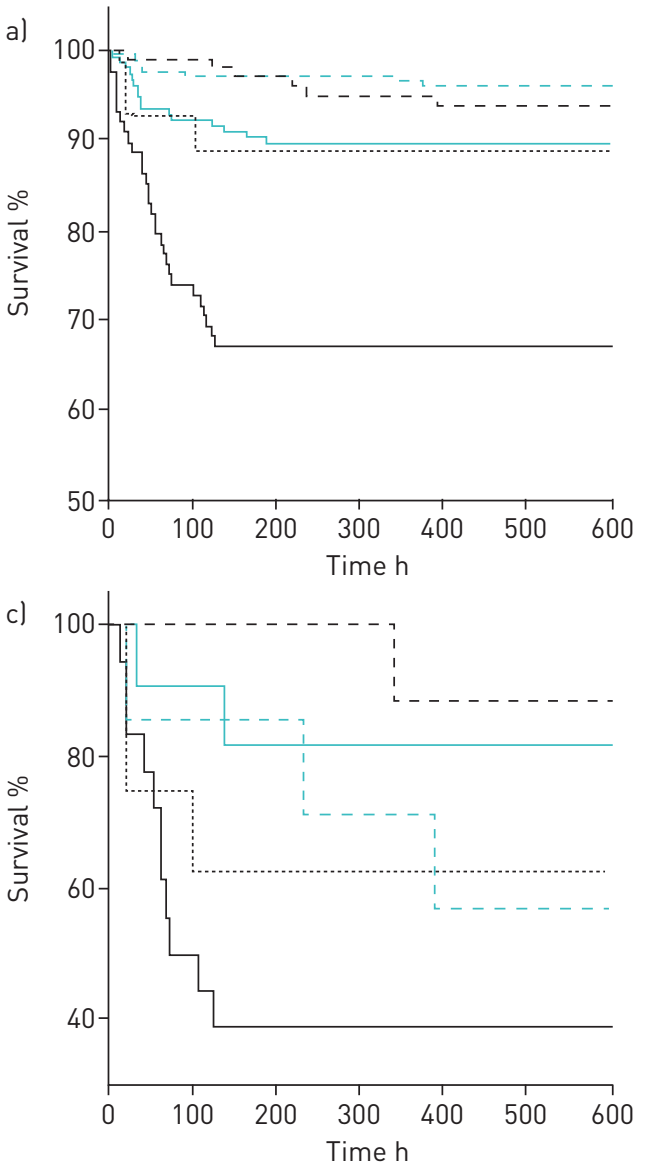

b)

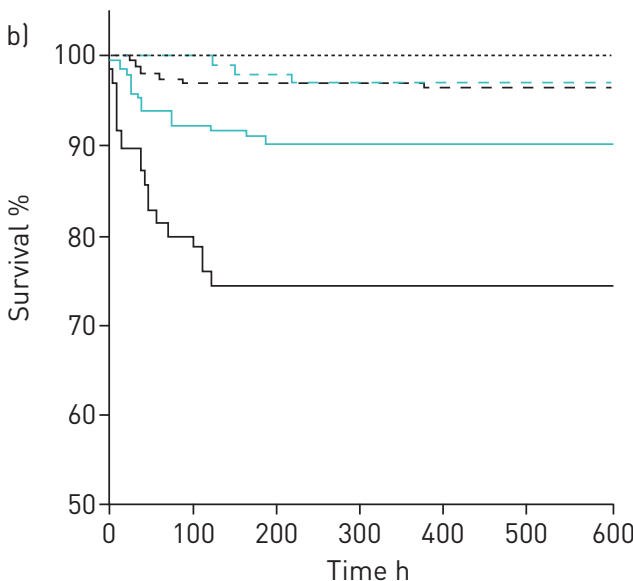

Mask-on proportion:

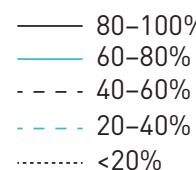

FIGURE 4 Survival stratified by type of respiratory failure and mask-on proportion (mask-on time relative to total therapy duration) calculated as mask-on time/(mask-on time+mask-off timel representing noninvasive ventilation "intensity". a) All patients, b) hypercapnic respiratory failure patients and c) hypoxaemic respiratory failure patients.

In summary, we have demonstrated a dose-response relationship between the duration of direct NIV use (mask-on time) in acute respiratory failure and hospital survival up to $24 \mathrm{~h}$ cumulative usage in HCRF. Benefits in survival are associated with as little as $2 \mathrm{~h}$ of this therapy. There is an association between reduced survival and increased proportion of usage in both HCRF and HRF, most likely reflecting underlying acuity of disease and dependence on ventilation.

Conflict of interest: None declared.

\section{References}

1 Cabrini L, Landoni G, Oriani A, et al. Noninvasive ventilation and survival in acute care settings: a comprehensive systematic review and metaanalysis of randomized controlled trials. Crit Care Med 2015; 43: 880-888.

2 Nava S, Hill N. Non-invasive ventilation in acute respiratory failure. Lancet 2009; 374: 250-259.

3 Plant PK, Owen JL, Elliott MW. Early use of non-invasive ventilation for acute exacerbations of chronic obstructive pulmonary disease on general respiratory wards: a multicentre randomised controlled trial. Lancet 2000; 355: 1931-1935.

4 Kramer N, Meyer TJ, Meharg J, et al. Randomized, prospective trial of noninvasive positive pressure ventilation in acute respiratory failure. Am J Respir Crit Care Med 1995; 151: 1799-1806.

5 Lemiale V, Mokart D, Resche-Rigon M, et al. Effect of noninvasive ventilation vs oxygen therapy on mortality among immunocompromised patients with acute respiratory failure: a randomized clinical trial. JAMA 2015; 314 $1711-1719$.

6 Nava S, Grassi M, Fanfulla F, et al. Non-invasive ventilation in elderly patients with acute hypercapnic respiratory failure: a randomised controlled trial. Age Ageing 2011; 40: 444-450.

7 Hilbert G, Gruson D, Vargas F, et al. Noninvasive ventilation in immunosuppressed patients with pulmonary infiltrates, fever, and acute respiratory failure. N Engl J Med 2001; 344: 481-487.

8 Gursel G, Aydogdu M, Tasyurek S, et al. Factors associated with noninvasive ventilation response in the first day of therapy in patients with hypercapnic respiratory failure. Ann Thorac Med 2012; 7: 92-97.

9 Kleopa KA, Sherman M, Neal B, et al. Bipap improves survival and rate of pulmonary function decline in patients with ALS. J Neurol Sci 1999; 164: 82-88. 
10 Aboussouan LS, Khan SU, Banerjee M, et al. Objective measures of the efficacy of noninvasive positive-pressure ventilation in amyotrophic lateral sclerosis. Muscle Nerve 2001; 24: 403-409.

11 Castro-Anon O, Perez de Llano A, De la Fuente Sanchez S, et al. Obesity-hypoventilation syndrome: increased risk of death over sleep apnea syndrome. PLoS One 2015; 10: e0117808.

12 Nickol AH, Hart N, Hopkinson NS, et al. Mechanisms of improvement of respiratory failure in patients with restrictive thoracic disease treated with non-invasive ventilation. Thorax 2005; 60: 754-760.

13 Sorbo LD, Martin E, Ranieri VM Hypoxic respiratory failure. In: Mason RJ, Broaddus VC, Martin TR, et al., eds. Murray and Nadel's Textbook of Respiratory Medicine. 5 edn. Philadelphia, Saunders, 2010; pp. 2130-2137.

14 Brochard L, Mancebo J, Wysocki M, et al. Noninvasive ventilation for acute exacerbations of chronic obstructive pulmonary disease. $N$ Engl J Med 1995; 333: 817-822.

15 Bott J, Carroll MP, Conway JH, et al. Randomised controlled trial of nasal ventilation in acute ventilatory failure due to chronic obstructive airways disease. Lancet 1993; 341: 1555-1557.

16 Wysocki M, Antonelli M. Noninvasive mechanical ventilation in acute hypoxaemic respiratory failure. Eur Respir J 2001; 18: 209-220.

17 Agarwal R, Aggarwal AN, Gupta D. Role of noninvasive ventilation in acute lung injury/acute respiratory distress syndrome: a proportion meta-analysis. Respir Care 2010; 55: 1653-1660.

18 Vianello A, Arcaro G, Battistella L, et al. Noninvasive ventilation in the event of acute respiratory failure in patients with idiopathic pulmonary fibrosis. J Crit Care 2014; 29: 562-567.

19 Neuschwander A, Lemiale V, Darmon M, et al. Noninvasive ventilation during acute respiratory distress syndrome in patients with cancer: trends in use and outcome. J Crit Care 2017; 38: 295-299.

20 Carrillo A, Ferrer M, Gonzalez-Diaz G, et al. Noninvasive ventilation in acute hypercapnic respiratory failure caused by obesity hypoventilation syndrome and chronic obstructive pulmonary disease. Am J Respir Crit Care Med 2012; 186: 1279-1285.

21 Miller D, Fraser K, Murray I, et al. Predicting survival following non-invasive ventilation for hypercapnic exacerbations of chronic obstructive pulmonary disease. Int J Clin Pract 2012; 66: 434-437.

22 Antonelli M, Conti G, Moro ML, et al. Predictors of failure of noninvasive positive pressure ventilation in patients with acute hypoxemic respiratory failure: a multi-center study. Intensive Care Med 2001; 27: 1718-1728. 